KYUNGPOOK Math. J. 54(2014), 293-320

http://dx.doi.org/10.5666/KMJ.2014.54.2.293

\title{
Global Chaos Synchronization of WINDMI and Coullet Chaotic Systems using Adaptive Backstepping Control Design
}

\author{
SuREsh RAsappan* And Sundarapandian VAIDyanathan \\ Department of Mathematics, Vel Tech University, \# 42 Avadi - Vel Tech Road, \\ Avadi, Chennai, Tamilnadu, India, 600062 \\ e-mail : mrpsuresh83@gmail.com and sundarvtu@gmail.com
}

ABstract. In this paper, global chaos synchronization is investigated for WINDMI (J. C. Sprott, 2003) and Coullet (P. Coullet et al, 1979) chaotic systems using adaptive backstepping control design based on recursive feedback control. Our theorems on synchronization for WINDMI and Coullet chaotic systems are established using Lyapunov stability theory. The adaptive backstepping control links the choice of Lyapunov function with the design of a controller and guarantees global stability performance of strict-feedback chaotic systems. The adaptive backstepping control maintains the parameter vector at a predetermined desired value. The adaptive backstepping control method is effective and convenient to synchronize and estimate the parameters of the chaotic systems. Mainly, this technique gives the flexibility to construct a control law and estimate the parameter values. Numerical simulations are also given to illustrate and validate the synchronization results derived in this paper.

\section{Introduction}

Chaos refers to one type of complex dynamical behaviors that possess extreme sensitivity to tiny variations of initial conditions, bounded trajectories in phase space and fractional topological dimensions. In general, synchronization research has been focused on two areas. The first one works with the state observers, where the main applications pertain to the synchronization of nonlinear oscillators. The second one is the use of control laws, which allows to achieve the synchronization between nonlinear oscillators, with different structures and orders.

The synchronization of chaotic system was first researched by Yamada and Fujisaka [1] with subsequent work by Pecora and Carroll $[2,3]$. The synchronization of chaos is one way of explaining sensitive dependence on initial conditions. It has

* Corresponding Author.

Received August 1, 2012; revised June 3, 2013; accepted July 26, 2013.

2010 Mathematics Subject Classification: 34H10, 93C15, 34H15.

Key words and phrases: Chaos, Synchronization, Adaptive Backstepping Control, WINDMI System, Coullet System. 
been established that the synchronization of two chaotic systems, that identify the tendency of two or more systems are coupled together to undergo closely related motions. The problem of chaos synchronization is to design a coupling between the two systems such that the chaotic time evaluation becomes ideal. The output of the slave system asymptotically follows the output of the master system i.e. the output of the master system controls the output of the slave system.

The synchronization for chaotic systems has been widespread to the scope, such as generalized synchronization [6], phase synchronization [7], lag synchronization, projective synchronization [8], generalized projective synchronization $[9,10,11,12]$ and even anti-synchronization $[13,14]$. A variety of schemes for ensuring the control and synchronization of such systems have been demonstrated based on their potential applications in various fields including chaos generator design, secure communication [15, 16], physical systems [17], and chemical reaction [18], ecological systems [19], information science [20], energy resource systems [21], ghostburster neurons [22], bi-axial magnet models [23], neuronal models [24, 25], SIR epidemic models with impulsive vaccination [26] and predicting the influence of solar wind to celestial bodies [27, 28, 29, 30], etc. So far a variety of impressive approaches have been proposed for the synchronization of the chaotic systems such as the OGY method[31], sampled feedback synchronization method, time delay feedback method [32], sliding mode control method [33, 34], active control method [35], backstepping control [37, 38, 39, 40, 41] etc.

Adaptive backstepping control design is a direct aggregation of a control methodology with some form of a recursive system identification and the system identification could be aimed to determining the system to be controlled is linear or nonlinear systems. The system identification is only the parameters of a fixed type of model that need to be determined and limiting the parametric system identification and parametric adaptive control [42, 43, 44, 45, 46, 47]. Adaptive backstepping control design is studied and analyzed in theory of unknown but fixed parameter systems.

In this paper, adaptive backstepping control design with feedback input approach has been proposed. This approach is a systematic design approach and guarantees global stability of the WINDMI (J. C. Sprott, [48]) and Coullet (P. Coullet et al, [49]) chaotic systems. Based on the Lyapunov function, the adaptive update control is determined to tune the controller gain based on the precalculated feedback control inputs. We organize this paper as follows. In Section 2, we present the methodology of chaos synchronization by adaptive backstepping control method. In Section 3, we give a description of the chaotic systems discussed in this paper. In Section 4, we demonstrate the chaos synchronization of identical WINDMI chaotic system using adaptive backstepping control. In Section 5, we demonstrate the chaos synchronization of identical Coullet chaotic system using adaptive backstepping control. In Section 6, we demonstrate the chaos synchronization of WINDMI and Coullet chaotic system using adaptive backstepping control. In Section 7, we summarize the results obtained in this paper. 


\section{Problem Statement and Our Methodology}

In general, the two dynamic systems in synchronization are called the master and slave system respectively. A well designed controller will make the trajectory of the slave system track the trajectory of the master system, that is, the two systems will be synchronous.

Consider the master system described by the dynamics

$$
\begin{aligned}
\dot{x}_{1} & =F_{1}\left(x_{1}, x_{2}, \ldots, x_{n}, \alpha_{i}\right), \\
\dot{x}_{2} & =F_{2}\left(x_{1}, x_{2}, \ldots, x_{n}, \alpha_{i}\right), \\
\dot{x}_{3} & =F_{3}\left(x_{1}, x_{2}, \ldots, x_{n}, \alpha_{i}\right), \\
\vdots & \vdots \\
\dot{x}_{n} & =F_{n}\left(x_{1}, x_{2}, \ldots, x_{n}, \alpha_{i}\right),
\end{aligned}
$$

where $x(t) \in \mathbb{R}^{n}$ is a state vectors of the system and and $\alpha_{i}$ are positive unknown parameters, $\hat{\alpha}_{i}$ are estimates of the parameters $\alpha_{i}$.

Consider the slave system with the controller $u$ described by the dynamics

$$
\begin{aligned}
\dot{y}_{1} & =G_{1}\left(y_{1}, y_{2}, \ldots, y_{n}, \alpha_{i}\right) \\
\dot{y}_{2} & =G_{2}\left(y_{1}, y_{2}, \ldots, y_{n}, \alpha_{i}\right) \\
\dot{y}_{3} & =G_{3}\left(y_{1}, y_{2}, \ldots, y_{n}, \alpha_{i}\right) \\
\vdots & \vdots \\
\dot{y}_{n} & =G_{n}\left(y_{1}, y_{2}, \ldots, y_{n}, \alpha_{i}\right)+u(t),
\end{aligned}
$$

where $u$ is the input to the system with parameter estimator $\hat{\alpha}_{i}, i=1,2,3, \ldots, n$, $y \in \mathbb{R}^{n}$ is state of the system and $F_{i}, G_{i}(i=1,2,3, \ldots, n)$ are linear and nonlinear functions with inputs from systems (2.1) and (2.2).

If $F_{i}$ equals to $G_{i}$, then the systems states are identical synchronization otherwise that systems states are non identical chaotic synchronization of systems. The chaotic systems (2.1) and (2.2) depend not only on state variables but also on time $\mathrm{t}$ and the parameters.

We define the synchronization error as

$$
e=y_{i}-x_{i}
$$

then the error dynamics is obtained as

$$
\begin{aligned}
\dot{e}_{1} & =G_{1}\left(y_{1}, y_{2}, \ldots, y_{n}, \alpha_{i}\right)-F_{1}\left(x_{1}, x_{2}, \ldots, x_{n}, \alpha_{i}\right) \\
\dot{e}_{2} & =G_{2}\left(y_{1}, y_{2}, \ldots, y_{n}, \alpha_{i}\right)-F_{2}\left(x_{1}, x_{2}, \ldots, x_{n}, \alpha_{i}\right), \\
\dot{e}_{3} & =G_{3}\left(y_{1}, y_{2}, \ldots, y_{n}, \alpha_{i}\right)-F_{3}\left(x_{1}, x_{2}, \ldots, x_{n}, \alpha_{i}\right) \\
\vdots & \vdots \\
\dot{e}_{n} & =G_{n}\left(y_{1}, y_{2}, \ldots, y_{n}, \alpha_{i}\right)-F_{n}\left(x_{1}, x_{2}, \ldots, x_{n}, \alpha_{i}\right)+u(t),
\end{aligned}
$$


where $u$ is the controller to the system with parameter estimator $\hat{\alpha}_{i}$. The parameter estimation error is defined as

$$
e_{\alpha_{i}}=\alpha_{i}-\hat{\alpha}_{i}, i=1,2,3, \ldots, n \text {. }
$$

The synchronization error system controls a controlled chaotic system with control input $u$ with adaptive update law $\dot{\hat{\alpha}}_{i}$ as a function of the parameter estimator error states $e_{\alpha_{1}}, e_{\alpha_{2}}, e_{\alpha_{3}}, \ldots, e_{\alpha_{n}}$. That means the systematic adaptive feedbacks so as to stabilize the error dynamics $(2.3), e_{1}, e_{2}, e_{3}, \ldots, e_{n}$ converge to zero as time t tends to infinity. This implies that the controller $u$ and adaptive update law $\dot{\hat{\alpha}}_{i}$ should be designed so that the two chaotic systems can be synchronized. In mathematically

$$
\lim _{t \rightarrow \infty}\|e(t)\|=0 .
$$

Adaptive backstepping control design is systematic and guarantees global stability performance of strict-feedback chaotic systems. By using the adaptive backstepping control design, the chaotic system is stabilized with respect to a Lyapunov function $V$, by the design of parameter estimator control $\hat{\alpha}_{i}$ and a control input function $u_{i}$ with adaptive update law $\dot{\hat{\alpha}}_{i}$. The Lyapunov stability approach consists in finding an update law. We use Lyapunov function technique as our methodology.

We consider the stability of the system

$$
\dot{e}_{1}=G_{1}\left(y_{1}, y_{2}, \ldots, y_{n}, \alpha_{i}\right)-F_{1}\left(x_{1}, x_{2}, \ldots, x_{n}, \alpha_{i}\right),
$$

where the state variables $x(t) \in \mathbb{R}^{n}, y(t) \in \mathbb{R}^{n}$. As long as this feedback stabilize the system (2.4) converge to zero as the time t goes to infinity, where $e_{2}=\beta_{1}\left(e_{1}\right)$ regarded as an virtual controller. For the design of $\beta_{1}\left(e_{1}\right)$ to stabilize the subsystem (2.4).

We consider the Lyapunov function defined by

$$
V_{1}\left(e_{1}, e_{\alpha_{i}}\right)=e_{1}^{T} P_{1} e_{1}+\sum_{i=1}^{i=k} e_{\alpha_{i}}^{T} Q_{1} e_{\alpha_{i}},
$$

where $P_{1}$ and $Q_{1}$ are positive definite matrices.

Suppose the derivative of $V_{1}$ is

$$
\dot{V}_{1}=-e_{1}^{T} R_{1} e_{1}-\sum_{i=1}^{i=k} e_{\alpha_{i}}^{T} S_{1} e_{\alpha_{i}},
$$

where $R_{1}$ and $S_{1}$ are positive definite matrices.

Then $\dot{V}_{1}$ is negative definite function.

Thus by Lyapunov stability theory [50] the error dynamics (2.4) is globally asymptotically stable.

The virtual control $e_{2}=\beta_{1}\left(e_{1}\right)$ makes the system (2.4) asymptotically stable. The function $\beta_{1}\left(e_{1}\right)$ is estimative when $e_{2}$ considered as controller. 
The error between $e_{2}$ and $\beta_{1}\left(e_{1}\right)$ is

$$
w_{2}=e_{2}-\beta_{1}\left(e_{1}\right),
$$

Consider the $\left(e_{1}, w_{2}\right)$ subsystem given by

$$
\begin{aligned}
\dot{e}_{1} & =G_{1}\left(y_{1}, y_{2}, \ldots, y_{n}, e_{\alpha_{i}}\right)-F_{1}\left(x_{1}, x_{2}, \ldots, x_{n}, e_{\alpha_{i}}\right), \\
\dot{w}_{2} & =G_{2}\left(y_{1}, y_{2}, \ldots, y_{n}, e_{\alpha_{i}}\right)-F_{2}\left(x_{1}, x_{2}, \ldots, x_{n}, e_{\alpha_{i}}\right)-\dot{\beta}_{1}\left(e_{1}\right) .
\end{aligned}
$$

Let $e_{3}$ be a virtual controller in system (2.8). Assume that when

$$
e_{3}=\beta_{2}\left(e_{1}, w_{2}\right)
$$

the system (2.8) made globally asymptotically stable.

Consider the Lyapunov function defined by

$$
V_{2}\left(e_{1}, w_{2}\right)=V_{1}\left(e_{1}\right)+w_{2}^{T} P_{2} w_{2}+\sum_{i=k+1}^{i=m} e_{\alpha_{i}}^{T} Q_{2} e_{\alpha_{i}},
$$

where $P_{2}$ and $S_{2}$ are positive definite matrices.

Suppose the derivative of $V_{2}$ is

$$
\dot{V}_{2}=-e_{1}^{T} R_{1} e_{1}-w_{2}^{T} R_{2} w_{2}-\sum_{i=k+1}^{i=m} e_{\alpha_{i}}^{T} S_{2} e_{\alpha_{i}} .
$$

where $R_{1}, R_{2}, S_{2}$ are positive definite matrices.

Then $\dot{V}_{2}$ is a negative definite function.

Thus by Lyapunov stability theory the error dynamics (2.10) is globally asymptotically stable.

The virtual control is $e_{2}=\beta_{2}\left(e_{1}, w_{2}\right)$ and it make the system (2.10) globally asymptotically stable.

For the $n^{\text {th }}$ state of the error dynamics, define the error variable $w_{n}$ as

$$
w_{n}=e_{n}-\beta_{n-1}\left(e_{1}, w_{2}, w_{3}, \ldots, w_{n}\right) .
$$

Consider the $\left(e_{1}, w_{2}, w_{3}, \ldots, w_{n}\right)$ subsystem given by

$$
\begin{aligned}
\dot{e}_{1}= & G_{1}\left(y_{1}, y_{2}, \ldots, y_{n}, e_{\alpha_{i}}\right)-F_{1}\left(x_{1}, x_{2}, \ldots, x_{n}, e_{\alpha_{i}}\right), \\
\dot{w_{2}}= & G_{2}\left(y_{1}, y_{2}, \ldots, y_{n}, e_{\alpha_{i}}\right)-F_{2}\left(x_{1}, x_{2}, \ldots, x_{n}, e_{\alpha_{i}}\right)-\dot{\beta}_{1}\left(e_{1}\right), \\
\vdots & \vdots \\
\dot{w_{n}}= & G_{n}\left(y_{1}, y_{2}, \ldots, y_{n}, e_{\alpha_{i}}\right)-F_{n}\left(x_{1}, x_{2}, \ldots, x_{n}, e_{\alpha_{i}}\right) \\
& -\dot{\beta}_{n-1}\left(e_{1}, w_{2}, w_{3}, \ldots, w_{n}\right)+u_{t} .
\end{aligned}
$$

Consider the Lyapunov function defined by

$$
V_{n}\left(e_{1}, w_{2}, w_{3}, \ldots, w_{n}, e_{\alpha_{i}}\right)=V_{n-1}\left(e_{1}, w_{2}, w_{3}, \ldots, w_{n-1}\right)+w_{n}^{T} P_{n} w_{n}+\sum_{i=m+1}^{i=n} e_{\alpha_{i}}^{T} Q_{n} e_{\alpha_{i}}
$$


where $P_{n}$ and $Q_{n}$ are positive definite matrices.

Suppose the derivative of $V_{n}$ is

$$
\dot{V}_{n}=-e_{1}^{T} R_{1} e_{1}-w_{2}^{T} R_{2} w_{2}-\ldots .-w_{n}^{T} R_{n} w_{n}-\sum_{i=m+1}^{n} e_{\alpha_{i}}^{T} S_{n} e_{\alpha_{i}},
$$

where $R_{1}, R_{2}, R_{3}, \ldots, R_{n}$ and $S_{n}$ are positive definite matrices.

Then $\dot{V}_{n}$ is a negative definite function on $\mathbb{R}^{n}$.

Thus by Lyapunov stability theory the error dynamics (2.13) is globally asymptotically stable.

The virtual control is $e_{n}=\beta_{n-1}\left(e_{1}, w_{2}, w_{2}, \ldots, w_{n-1}\right)$ and the state feedback input $u$ makes the system (2.13) globally asymptotically stable.

Hence, the states of the master and slave systems are globally and asymptotically synchronized and the adaptive control law is given by

$$
\dot{\hat{\alpha}}_{i}=G(e)+k_{i} e_{\alpha_{i}}
$$

where $k_{i}$ is positive constant, $e=y-x$ is the error vector, and $G: \mathbb{R}^{n} \rightarrow \mathbb{R}^{n}$ is a continuous vector function with the error as its argument.

\section{The System Description}

Recently, theoretical design and hardware implementation of different kinds of chaotic oscillators have attracted increasing attention, aiming real world applications of many chaos based technologies and information systems.

\subsection{The WINDMI system}

The solar-wind driven magnetosphere-ionosphere is a complex driven-damped dynamical system. The system gives variety of dynamical properties such as low-level steady plasma conversion, quasiperiodic releases of geotail stored plasma energy into the ionosphere, states of continuous strong unloading provisionally identified as magnetic stromes.

When the prediction of whether modeling, errors in initial values of the input data, chaotic flucations in the forcing functions and internal chaotic dynamics from the nonlinearity and feedback loops in the deterministic systems are. The WINDMI model is well accepted mathematical model for solar-wind-driven magnetosphereionosphere system. WINDMI model is directly track energy through the global magnetosphere. The global energy properties of substrome using measured magnetosphere data is drive the WINDMI model.

The WINDMI (J. C. Sprott, [48]) system is a complex driven-damped dynamical system. The WINDMI system describes as the energy flow through the solar wind magnetosphere-ionosphere system. The dynamics of the chaotic WINDMI system 
is described by

$$
\begin{aligned}
& \dot{x}_{1}=x_{2}, \\
& \dot{x}_{2}=x_{3}, \\
& \dot{x}_{3}=-a x_{3}-x_{2}+b-e^{x_{1}},
\end{aligned}
$$

where $x_{1}, x_{2}, x_{3}$ are state variables and $a, b$ are positive real constants. The WINDMI system (3.1.1) is chaotic when

$$
a=0.7, b=1.5
$$

Fig. 1 depicts the WINDMI chaotic attractor.

WINDMI model enable to estimate the dimensionless parameter values for the planets with magnetospheres. This model gives a useful perspective on the stome and substrom problem. In geotail, there existing micro instabilities of collisionless tearing modes and kinetic ballooning modes, WINDMI model could be used as an external frame work for that modes.

\subsection{The Coullet system}

The Coullet (P. Coullet et al, [49]) chaotic systems, proposed by Coullet and Arneodo. The Coullet chaotic system is one of the paradigms of chaotic system and it includes a simple cubic part and three positive parameters. The dynamics of the chaotic Coullet system is described by

$$
\begin{aligned}
& \dot{x}_{1}=x_{2}, \\
& \dot{x}_{2}=x_{3}, \\
& \dot{x}_{3}=a x_{1}-b x_{2}-c x_{3}-x_{1}^{3},
\end{aligned}
$$

where $x_{1}, x_{2}, x_{3}$ are state variables and $a, b$ and $c$ are positive real constants. The Coullet system (3.2.1) is chaotic when

$$
a=5.5, b=3.5, \text { and } c=1
$$

Fig. 2 depicts the Coullet chaotic attractor.

\section{Synchronization of Identical WINDMI Chaotic Systems using Adap- tive Backstepping Control Design}

In this section we apply the adaptive backstepping method for the synchronization of two identical WINDMI chaotic systems (J. C. Sprott, [48]) when the parameter values are unknown. Thus, the master system is described by the chaotic WINDMI dynamics

$$
\begin{aligned}
& \dot{x}_{1}=x_{2}, \\
& \dot{x}_{2}=x_{3}, \\
& \dot{x}_{3}=-a x_{3}-x_{2}+b-e^{x_{1}},
\end{aligned}
$$


where $x_{1}, x_{2}, x_{3}$ are state variables and $a, b$ are positive unknown parameters, $\hat{a}$ and $\hat{b}$ are estimates of the parameters $a$ and $b$.

The slave system is also described by the chaotic WINDMI dynamics

$$
\begin{aligned}
& \dot{y}_{1}=y_{2}, \\
& \dot{y}_{2}=y_{3}, \\
& \dot{y}_{3}=-a y_{3}-y_{2}+b-e^{y_{1}}+u,
\end{aligned}
$$

where $y_{1}, y_{2}, y_{3}$ are state variables and $u$ is the backstepping controller to be designed.

The synchronization error is defined by

$$
e_{1}=y_{1}-x_{1}, e_{2}=y_{2}-x_{2}, e_{3}=y_{3}-x_{3} .
$$

The error dynamics is obtained as

$$
\begin{aligned}
& \dot{e}_{1}=e_{2}, \\
& \dot{e}_{2}=e_{3}, \\
& \dot{e}_{3}=-a e_{3}-e_{2}-e^{y_{1}}+e^{x_{1}}+u .
\end{aligned}
$$

The objective is to find the control law and adaptive update law, so that the system (4.3) is asymptotically stabilized at the origin and estimates the unknown parameters $a$ and $b$. We introduce the backstepping procedure to design the controller $u$, where $u$ is control feedback, as long as these feedback stabilize system (4.3) converge to zero as the time $t \rightarrow \infty$.

First we consider the stability of the system

$$
\dot{e}_{1}=e_{2},
$$

where $e_{2}$ is regarded as virtual controller.

We consider the Lyapunov function defined by

$$
V_{1}\left(e_{1}\right)=\frac{1}{2} e_{1}^{2} .
$$

The derivative of $V_{1}$ is as following

$$
\dot{V}_{1}=e_{1} e_{2} .
$$

Assume the controller $e_{2}=\beta_{1}\left(e_{1}\right)$.

If we choose

$$
\beta_{1}\left(e_{1}\right)=-k_{1} e_{1},
$$

then

$$
\dot{V}_{1}=-k_{1} e_{1}^{2},
$$


which is negative definite.

Hence the system (4.4) asymptotically stable.

The function $\beta_{1}\left(e_{1}\right)$ is an estimative function when $e_{2}$ is considered as a controller.

The error between $e_{2}$ and $\beta_{1}\left(e_{1}\right)$ is

$$
w_{2}=e_{2}-\beta_{1}\left(e_{1}\right) \text {. }
$$

Consider the $\left(e_{1}, w_{2}\right)$ subsystem given by

$$
\begin{gathered}
\dot{e}_{1}=w_{2}-k_{1} e_{1}, \\
\dot{w}_{2}=e_{3}+k_{1} e_{2} .
\end{gathered}
$$

Let $e_{3}$ be a virtual controller in system (4.10), assume when $e_{3}=\beta_{2}\left(e_{1}, w_{2}\right)$, the system (4.10)is made globally asymptotically stable.

Consider the Lyapunov function defined by

$$
V_{2}\left(e_{1}, w_{2}\right)=V_{1}\left(e_{1}\right)+\frac{1}{2} w_{2}^{2} .
$$

The derivative of $V_{2}\left(e_{3}, w_{2}\right)$ is

$$
\dot{V}_{2}=-k_{1} e_{1}^{2}+w_{2}\left(e_{1}+e_{3}+k_{1} w_{2}-k_{1}^{2} e_{1}\right) .
$$

We choose

$$
\beta_{2}\left(e_{1}, w_{2}\right)=-e_{1}-k_{1} w_{2}+k_{1}^{2} e_{1}-k_{2} w_{2} .
$$

Then it follows that

$$
\dot{V}_{2}=-k_{1} e_{1}^{2}-k_{2} w_{2}^{2} .
$$

Thus, $\dot{V}_{2}$ is negative definite function and hence the system (4.10) is globally asymptotically stable.

The error between $e_{3}$ and $\beta_{2}\left(e_{1}, w_{2}\right)$ is

$$
w_{3}=e_{3}-\beta_{2}\left(e_{1}, w_{2}\right) .
$$

Consider the $\left(e_{1}, w_{2}, w_{3}\right)$ subsystem given by

$$
\begin{aligned}
\dot{e}_{1}= & w_{2}-k_{1} e_{1}, \\
\dot{w}_{2}= & w_{3}-e_{1}-k_{2} w_{2}, \\
\dot{w}_{3}= & -a w_{3}+a e_{1}+a k_{1} w_{2}-a k_{1}^{2} e_{1}-e^{y_{1}}+e^{x_{1}}-\left(k_{1}+k_{2}\right) e_{1} \\
& +\left(k_{1}+k_{2}\right) w_{3}-\left(k_{1}^{2}+k_{2}^{2}\right) w_{2}+k_{1}^{3} e_{1}-k_{1} k_{2} w_{2}+u .
\end{aligned}
$$

Consider the Lyapunov function defined by

$$
V_{3}\left(e_{1}, w_{2}, w_{3}, e_{a}, e_{b}\right)=V_{2}\left(e_{1}, w_{2}\right)+\frac{1}{2} w_{3}^{2}+\frac{1}{2} e_{a}^{2}+\frac{1}{2} e_{b}^{2}
$$


Let us define the parameter estimation error as

$$
e_{a}=a-\hat{a}, e_{b}=b-\hat{b}
$$

Differentiating equation (4.17) along the trajectories (4.18) and using

$$
\dot{e_{a}}=-\dot{\hat{a}}, \dot{e_{b}}=-\dot{\hat{b}}
$$

The derivative of $V_{3}\left(e_{1}, w_{2}, w_{3}, e_{a}, e_{b}\right)$ is

$$
\begin{aligned}
\dot{V}_{3}= & -k_{1} e_{1}^{2}-k_{2} w_{2}^{2}-w_{3}\left[-a w_{3}+a e_{1}+a k_{1} w_{2}-a k_{1}^{2} e_{1}+a k_{2} w_{2}\right. \\
& -e^{y_{1}}+e^{x_{1}}-\left(k_{1}+k_{2}\right) e_{1}+\left(k_{1}+k_{2}\right) w_{3}-\left(k_{1}^{2}-k_{2}^{2}\right) w_{2}+k_{1}^{3} e_{1} \\
& \left.-k_{1} k_{2} w_{2}+u\right]+e_{a}(-\dot{\hat{a}})+e_{b}(-\hat{\hat{b}}) .
\end{aligned}
$$

We choose the controller $u$ as follows

$$
\begin{aligned}
u= & -w_{2}+\hat{a} w_{3}-\hat{a} e_{1}-\hat{a} k_{1} w_{2}+\hat{a} k_{1}^{2} e_{1}-\hat{a} k_{2} w_{2}+e^{y_{1}}-e^{x_{1}} \\
& -\left(k_{1}+k_{2}\right)\left(w_{3}-e_{1}\right)+\left(k_{1}^{2}-k_{2}^{2}\right) w_{2}-k_{1}^{3}+k_{1} k_{2} w_{2}-k_{3} w_{3}
\end{aligned}
$$

and the parameters are updated by the update law

$$
\begin{gathered}
\dot{\hat{a}}=w_{3}\left[e_{1}+k_{1} w_{2}-k_{1}^{2} e_{1}+k_{2} w_{2}-w_{3}\right]+k_{4} e_{a}, \\
\dot{\hat{b}}=k_{5} e_{b} .
\end{gathered}
$$

Then it follows that

$$
\dot{V}_{3}=-k_{1} e_{1}^{2}-k_{2} w_{2}^{2}-k_{3} w^{2}-k_{5} e_{a}^{2}-k_{6} e_{b}^{2} .
$$

Thus, $\dot{V}_{3}$ is negative definite function.

Thus by Lyapunov stability theory [50], the error dynamics (4.3) is globally asymptotically stable for all initial conditions.

Hence, the states of the master and slave systems are globally asymptotically synchronized.

Theorem 4.1. The identical WINDMI systems (4.1) and (4.2) are globally asymptotically synchronized with adaptive backstepping control

$$
\begin{aligned}
u= & -w_{2}+\hat{a} w_{3}-\hat{a} e_{1}-\hat{a} k_{1} w_{2}+\hat{a} k_{1}^{2} e_{1}-\hat{a} k_{2} w_{2}+e^{y_{1}}-e^{x_{1}} \\
& -\left(k_{1}+k_{2}\right)\left(w_{3}-e_{1}\right)+\left(k_{1}^{2}-k_{2}^{2}\right) w_{2}-k_{1}^{3}+k_{1} k_{2} w_{2}-k_{3} w_{3}
\end{aligned}
$$

by using adaptive parameter update law

$$
\begin{aligned}
& \dot{\hat{a}}=w_{3}\left[e_{1}+k_{1} w_{2}-k_{1}^{2} e_{1}+k_{2} w_{2}-w_{3}\right]+k_{4} e_{a}, \\
& \dot{\hat{b}}=k_{5} e_{b},
\end{aligned}
$$

where $k_{i}, i=1,2,3, \ldots 8$ are positive constants.

\subsection{Numerical simulation}


For the numerical simulations, the fourth order Runge-Kutta method is used to solve the system of differential equations (4.1) and (4.2) with the feedback controls $u$ given by (4.24). The parameters of the systems (4.1) and (4.2) are taken in the case of chaotic case as

$$
a=0.7, b=2.5
$$

The initial value of the master system (4.1) are chosen as

$$
x_{1}(0)=0.984, x_{2}(0)=0.345, x_{3}(0)=0.789
$$

and slave system (4.2) are chosen as

$$
y_{1}(0)=0.456, y_{2}(0)=0.812, y_{3}(0)=0.124
$$

The initial values of the estimated parameters are :

$$
\hat{a}(0)=10, \hat{b}=5.6 .
$$

We take the parameters $k_{i}=0.2, i=1,2,3 \ldots ., 8$.

Figure 3 depict the synchronization of identical WINDMI chaotic systems (4.1) and (4.2).

Figure 4 depict the synchronization error between identical WINDMI chaotic systems (4.1) and (4.2).

Figure 5 shows the estimated values of the parameters $\hat{a}$ and $\hat{b}$ converges to system parameters $a=0.7$ and $b=2.5$

\section{Synchronization of Identical Coullet Chaotic Systems using Adaptive Backstepping Control}

In this section we apply the adaptive backstepping method for the synchronization of two identical Coullet (P. Coullet et al, [49]) chaotic systems when the parameter values are unknown. Thus, the master system is described by the chaotic Coullet dynamics

$$
\begin{aligned}
& \dot{x}_{1}=x_{2}, \\
& \dot{x}_{2}=x_{3}, \\
& \dot{x}_{3}=a x_{1}-b x_{2}-c x_{3}-x_{1}^{3},
\end{aligned}
$$

where $x_{1}, x_{2}, x_{3}$ are state variables and $a, b, c$ are positive unknown parameters, $\hat{a}, \hat{b}$ and $\hat{c}$ are estimates of the parameters $a, b$ and $c$.

The slave system is also described by the chaotic Coullet dynamics

$$
\begin{aligned}
& \dot{y}_{1}=y_{2}, \\
& \dot{y}_{2}=y_{3}, \\
& \dot{y}_{3}=a y_{1}-b y_{2}-c y_{3}-y_{1}^{3}+u,
\end{aligned}
$$

where $y_{1}, y_{2}, y_{3}$ are state variables and $u$ is the backstepping controller to be designed. 
The synchronization error is defined by

$$
e_{1}=y_{1}-x_{1}, e_{2}=y_{2}-x_{2}, e_{3}=y_{3}-x_{3} .
$$

Then the error dynamics is obtained as

$$
\begin{aligned}
& \dot{e}_{1}=e_{2}, \\
& \dot{e}_{2}=e_{3}, \\
& \dot{e}_{3}=a e_{1}-b e_{2}-c e_{3}-y_{1}^{3}+x_{1}^{3}+u .
\end{aligned}
$$

The objective is to find the control law and adaptive update law, so that the system (5.4) is asymptotically stabilized at the origin and estimates the unknown parameters $a, b$ and $c$. We introduce the backstepping procedure to design the controller $u$, where $u$ is control feedback, as long as these feedback stabilize system (5.3) converge to zero as the time $t \rightarrow \infty$.

First we consider the stability of the system

$$
\dot{e}_{1}=e_{2},
$$

where $e_{2}$ is regarded as virtual controller.

We consider the Lyapunov function defined by

$$
V_{1}\left(e_{1}\right)=\frac{1}{2} e_{1}^{2} .
$$

The derivative of $V_{1}$ is as following

$$
\dot{V}_{1}=e_{1} e_{2}
$$

Assume the controller $e_{2}=\beta_{1}\left(e_{1}\right)$.

If we choose

$$
\beta_{1}\left(e_{1}\right)=-k_{1} e_{1},
$$

then

$$
\dot{V}_{1}=-k_{1} e_{1}^{2},
$$

which is negative definite function.

Hence the system (5.5) asymptotically stable.

Function $\beta_{1}\left(e_{1}\right)$ is an estimative function when $e_{2}$ is considered as a controller.

The error between $e_{2}$ and $\beta_{1}\left(e_{1}\right)$ is

$$
w_{2}=e_{2}-\beta_{1}\left(e_{1}\right)
$$

Consider the $\left(e_{1}, w_{2}\right)$ subsystem given by

$$
\begin{aligned}
& \dot{e}_{1}=w_{2}-k_{1} e_{1}, \\
& \dot{w}_{2}=e_{3}+k_{1} e_{2} .
\end{aligned}
$$


Let $e_{3}$ be a virtual controller in system (5.11).

Assume when $e_{3}=\beta_{2}\left(e_{1}, w_{2}\right)$, the system (5.11) made globally asymptotically stable.

Consider the Lyapunov function defined by

$$
V_{2}\left(e_{1}, w_{2}\right)=V_{1}\left(e_{1}\right)+\frac{1}{2} w_{2}^{2} .
$$

The derivative of $V_{2}\left(e_{3}, w_{2}\right)$ is

$$
\dot{V}_{2}=-k_{1} e_{1}^{2}+w_{2}\left(e_{1}+e_{3}+k_{1} w_{2}-k_{1}^{2} e_{1}\right) \text {. }
$$

We choose

$$
\beta_{2}\left(e_{1}\right)=-e_{1}-k_{1} w_{2}+k_{1}^{2} e_{1}-k_{2} w_{2} .
$$

Then it follows that

$$
\dot{V}_{2}=-k_{1} e_{1}^{2}-k_{2} w_{2}^{2} .
$$

Thus $\dot{V}_{2}$ is negative definite function.

Hence the system (5.11) is globally asymptotically stable.

Define the error between $e_{3}$ and $\beta_{2}\left(e_{1}, w_{2}\right)$ is

$$
w_{3}=e_{3}-\beta_{2}\left(e_{1}, w_{2}\right) .
$$

Consider the $\left(e_{1}, w_{2}, w_{3}\right)$ subsystem given by

$$
\begin{aligned}
\dot{e}_{1}= & w_{2}-k_{1} e_{1}, \\
\dot{w}_{2}= & w_{3}-e_{1}-k_{2} w_{2}, \\
\dot{w}_{3}= & a e_{1}-b e_{2}-c e_{3}-y_{1}^{3}+x_{1}^{3}+w_{2}-2 k_{1} e_{1}+\left(k_{1}+k_{2}\right) w_{3} \\
& -k_{1} k_{2} w_{2}+k_{1}^{3} e_{1}-k_{2} e_{1}-\left(k_{1}^{2}+k_{2}^{2}\right) w_{2} .
\end{aligned}
$$

Consider the Lyapunov function defined by

$$
V_{3}\left(e_{1}, w_{2}, w_{3}, e_{a}, e_{b}, e_{c}\right)=V_{2}\left(e_{3}, w_{2}\right)+\frac{1}{2} w_{3}^{2}+\frac{1}{2} e_{a}^{2}+\frac{1}{2} e_{b}^{2}+\frac{1}{2} e_{c}^{2}
$$

Let us define the parameter estimation error as

$$
e_{a}=a-\hat{a}, e_{b}=b-\hat{b}, e_{a}=c-\hat{c}
$$

Differentiating equation (5.18) along the trajectories (5.19) and using

$$
\dot{e_{a}}=-\dot{\hat{a}}, \dot{e_{b}}=-\dot{\hat{b}}, \dot{e_{c}}=-\dot{\hat{c}} .
$$

The derivative of $V_{3}\left(e_{1}, w_{2}, w_{3}, e_{a}, e_{b}, e_{c}\right)$ is

$$
\begin{aligned}
\dot{V}_{3}= & -k_{1} e_{1}^{2}-k_{2} w_{2}^{2}-w_{3}\left(a e_{1}-b e_{2}-c e_{3}-y_{1}^{3}+x_{1}^{3}+2 w_{2}\right. \\
& \left.-\left(2 k_{1}+k_{2}\right) e_{1}+\left(k_{1}+k_{2}\right) w_{3}-k_{1} k_{2} w_{2}+k_{1}^{3} e_{1}-\left(k_{1}^{2}+k_{2}^{2}\right) w_{2}+u_{3}\right) \\
& +e_{a}(-\dot{\hat{\hat{a}}})+e_{b}(-\hat{\hat{b}})+e_{c}(-\dot{\hat{c}}) .
\end{aligned}
$$


We choose the controller

$$
\begin{aligned}
u= & -\hat{a} e_{1}+\hat{b} e_{2}+\hat{c} e_{3}+y_{1}^{3}-x_{1}^{3}-2 w_{2}+\left(2 k_{1}+k_{2}\right) e_{1} \\
& -\left(k_{1}+k_{2}\right) w_{3}+k_{1} k_{2} w_{2}-k_{1}^{3}+\left(k_{1}^{2}+k_{2}^{2}\right) w_{2}-k_{3} w_{3}
\end{aligned}
$$

and the parameters are updated by the update law

$$
\begin{aligned}
\dot{\hat{a}} & =w_{3} e_{1}+k_{4} e_{a}, \\
\dot{\hat{b}} & =-w_{3} e_{2}+k_{5} e_{b} \text { and } \\
\dot{\hat{c}} & =-w_{3} e_{3}+k_{6} e_{c} .
\end{aligned}
$$

Then we obtain

$$
\dot{V}_{3}=-k_{1} e_{1}^{2}-k_{2} w_{2}^{2}-k_{3} w_{3}^{2}-k_{4} e_{a}^{2}-k_{5} e_{b}^{2}-k_{6} e_{c}^{2} .
$$

Thus $\dot{V}_{3}$ is negative definite function.

Thus by a Lyapunov stability theory [50], the error dynamics (5.3) is globally asymptotically stable for all initial conditions.

Hence, the states of the master and slave systems are globally and asymptotically synchronized.

Theorem 5.1. The Identical Coullet chaotic systems (5.1) and (5.2) are globally and asymptotically synchronized with adaptive backstepping control

$$
\begin{aligned}
u= & -\hat{a} e_{1}+\hat{b} e_{2}+\hat{c} e_{3}+y_{1}^{3}-x_{1}^{3}-2 w_{2}+\left(2 k_{1}+k_{2}\right) e_{1} \\
& -\left(k_{1}+k_{2}\right) w_{3}+k_{1} k_{2} w_{2}-k_{1}^{3}+\left(k_{1}^{2}+k_{2}^{2}\right) w_{2}-k_{3} w_{3},
\end{aligned}
$$

by using adaptive parameter update law

$$
\begin{aligned}
& \dot{\hat{a}}=w_{3} e_{1}+k_{4} e_{a}, \\
& \dot{\hat{b}}=-w_{3} e_{2}+k_{5} e_{b} \text { and } \\
& \dot{\hat{c}}=-w_{3} e_{3}+k_{6} e_{c},
\end{aligned}
$$

where $k_{i}, i=1,2,3, \ldots, 9$ are positive constants.

\subsection{Numerical simulation}

For the numerical simulations, the fourth order Runge-Kutta method is used to solve the differential equations (5.1) and (5.2) with the feedback controls $u$.

The parameters of the systems (5.1) and (5.2) are taken in the case of chaotic case as

$$
a=5.5, b=3.5, c=1 .
$$

The initial value of the drive system (5.1) are chosen as

$$
x_{1}(0)=0.125, x_{2}(0)=0.625, x_{3}(0)=0.825
$$

and response system (5.2) are chosen as

$$
y_{1}(0)=0.924, y_{2}(0)=0.498, y_{3}(0)=0.032
$$


The initial values of the parameter estimates are taken as:

$$
\hat{a}(0)=10, \hat{b}(0)=34.4, \hat{c}(0)=25 .
$$

We take the parameters $k_{i}=2, i=1,2,3 \ldots, 8$

Figure.6 depict the synchronization of identical Coullet chaotic systems (5.1) and (5.2).

Figure. 7 depict the synchronization error between identical Coullet chaotic systems (5.1) and (5.2).

Figure. 8 shows the estimated values of the parameters $\hat{a}, \hat{b}$ and $\hat{c}$ converges to system parameters $a=5.5, b=3.5$ and $c=1$.

\section{Synchronization of WINDMI and Coullet Chaotic Systems using Adaptive Backstepping Control Design}

In this section, the adaptive backstepping control design is applied for the synchronization of two different chaotic systems described by WINDMI (J. C. Sprott, [48]) system as the master system and Coullet (P. Coullet et al, [49]) system as the slave system. The dynamics of the WINDMI system, taken as master system is described by

$$
\begin{aligned}
& \dot{x}_{1}=x_{2}, \\
& \dot{x}_{2}=x_{3}, \\
& \dot{x}_{3}=-a x_{3}-x_{2}+b-e^{x_{1}},
\end{aligned}
$$

where $x_{1}, x_{2}, x_{3}$ are state variables, $a, b$ are positive unknown parameters, $\hat{a}$ and $\hat{b}$ are estimates of the parameters $a$ and $b$.

The dynamics of the Coullet system, taken as the slave system, is described by

$$
\begin{aligned}
& \dot{y}_{1}=y_{2}, \\
& \dot{y}_{2}=y_{3}, \\
& \dot{y}_{3}=\alpha y_{1}-\beta y_{2}-\gamma y_{3}-y_{1}^{3}+u,
\end{aligned}
$$

where $\alpha, \beta, \gamma$ are positive unknown parameters, $\hat{\alpha}, \hat{\beta}$ and $\hat{\gamma}$ are estimates of the parameters $\alpha, \beta$ and $\gamma . u$ is the backstepping controller to be designed so as to synchronize the states of the different chaotic systems (6.1) and (6.2).

The synchronization error is defined by

$$
e_{1}=y_{1}-x_{1}, e_{2}=y_{2}-x_{2}, e_{3}=y_{3}-x_{3} .
$$

The error dynamics is obtained as

$$
\begin{aligned}
& \dot{e}_{1}=e_{2}, \\
& \dot{e}_{2}=e_{3}, \\
& \dot{e}_{3}=\alpha y_{1}-\beta y_{2}-\gamma y_{3}-y_{1}^{3}+a x_{3}+x_{2}-b+e^{x_{1}}+u .
\end{aligned}
$$


The objective is to find the control law and adaptive update law, so that the system (6.4) is asymptotically stabilized at the origin and estimates the unknown parameters $a, b, c, \alpha, \beta$ and $\gamma$ We introduce the backstepping procedure to design the controller $u$, where $u$ is control feedback, as long as these feedback stabilize system (6.4) converge to zero as the time $t \rightarrow \infty$.

First we consider the stability of the system

$$
\dot{e}_{1}=e_{2},
$$

where $e_{2}$ is regarded as virtual controller.

We consider the Lyapunov function defined by

$$
V_{1}\left(e_{1}\right)=\frac{1}{2} e_{1}^{2} .
$$

The derivative of $V_{1}$ is as following

$$
\dot{V}_{1}=e_{1} e_{2} .
$$

Assume the virtual controller $e_{2}=\alpha_{1}\left(e_{1}\right)$.

If we choose

$$
\alpha_{1}\left(e_{1}\right)=-k_{1} e_{1},
$$

then

$$
\dot{V}_{1}=-k_{1} e_{1}^{2},
$$

which is a negative definite function.

Hence the system (6.4) asymptotically stable.

Function $\beta_{1}\left(e_{1}\right)$ is an estimative function when $e_{2}$ is considered as a controller.

The error between $e_{2}$ and $\beta_{1}\left(e_{1}\right)$ is

$$
w_{2}=e_{2}-\beta_{1}\left(e_{1}\right) .
$$

Consider $\left(e_{1}, w_{2}\right)$ subsystem given by

$$
\begin{aligned}
& \dot{e}_{1}=w_{2}-k_{1} e_{1}, \\
& \dot{w}_{2}=e_{3}+k_{1} w_{2}-k_{1}^{2} e_{1} .
\end{aligned}
$$

Let $e_{3}$ be a virtual controller in system (6.11).

Assume when $e_{3}=\beta_{2}\left(e_{1}, w_{2}\right)$, the system (6.11) is made globally asymptotically stable.

Consider the Lyapunov function defined by

$$
V_{2}\left(e_{1}, w_{2}\right)=V_{1}\left(e_{1}\right)+\frac{1}{2} w_{2}^{2} .
$$


The derivative of $V_{2}\left(e_{3}, w_{2}\right)$ is

$$
\dot{V}_{2}=-k_{1} e_{1}^{2}+w_{2}\left(e_{1}+e_{3}+k_{1} w_{2}-k_{1}^{2} e_{1}\right) .
$$

If we choose

$$
\beta_{2}\left(e_{1}\right)=-e_{1}-k_{1} w_{2}+k_{1}^{2} e_{1}-k_{2} w_{2}
$$

Then it follow that

$$
\dot{V}_{2}=-k_{1} e_{1}^{2}-k_{2} w_{2}^{2} .
$$

Thus, $\dot{V}_{2}$ is a negative definite function.

Hence the system (6.11)is globally asymptotically stable.

The error between $e_{3}$ and $\beta_{2}\left(e_{1}, w_{2}\right)$ is

$$
w_{3}=e_{3}-\beta_{2}\left(e_{1}, w_{2}\right) .
$$

Consider $\left(e_{1}, w_{2}, w_{3}\right)$ subsystem given by

$$
\begin{aligned}
\dot{e}_{1}= & w_{2}-k_{1} e_{1}, \\
\dot{w}_{2}= & w_{3}-e_{1}-k_{2} w_{2}, \\
\dot{w_{3}}= & \alpha y_{1}-\beta y_{2}-\gamma y_{3}-y_{1}^{3}+a x_{3}+x_{2}-b+e^{x_{1}}+w_{2}-\left(2 k_{1}+k_{2}\right) e_{1} \\
& +\left(k_{1}+k_{2}\right) w_{3}-k_{1} k_{2} w_{2}-\left(k_{1}^{2}+k_{2}^{2}\right) w_{2}+k_{1}^{3} e_{1}+u .
\end{aligned}
$$

Consider the Lyapunov function defined by

$$
\begin{aligned}
V_{3}\left(e_{1}, w_{2}, w_{3}, e_{\alpha}, e_{\beta}, e_{\gamma}, e_{a}, e_{b}\right)= & V_{2}\left(e_{3}, w_{2}\right)+\frac{1}{2} w_{3}^{2}+ \\
& \frac{1}{2} e_{\alpha}^{2}+\frac{1}{2} e_{\beta}^{2}+\frac{1}{2} e_{\gamma}^{2}++\frac{1}{2} e_{a}^{2}+\frac{1}{2} e_{b}^{2}
\end{aligned}
$$

Let us define the parameter estimation error as

$$
\begin{aligned}
e_{\alpha} & =\alpha-\hat{\alpha}, e_{\beta}=\beta-\hat{\beta}, e_{\gamma}=\gamma-\hat{\gamma} \\
e_{a} & =a-\hat{a}, e_{b}=b-\hat{b} .
\end{aligned}
$$

Differentiating equation (6.18) along the trajectories (6.19) and using

$$
\begin{aligned}
& \dot{e}_{\alpha}=-\dot{\hat{\alpha}}, \dot{e}_{\beta}=-\dot{\hat{\beta}}, \\
& \dot{e}_{\gamma}=-\dot{\hat{\gamma}}, \dot{e}_{a}=-\dot{\hat{a}}, \dot{e}_{b}=-\dot{\hat{b}} .
\end{aligned}
$$

The derivative of $V_{3}\left(e_{1}, w_{2}, w_{3}, e_{a}, e_{b}\right)$ is

$$
\begin{aligned}
\dot{V}_{3}= & -k_{1} e_{1}^{2}-k_{2} w_{2}^{2}-w_{3}\left[a e_{1}-b e_{2}-c e_{3}-y_{1}^{3}+x_{1}^{3}+2 w_{2}\right. \\
& \left.-\left(2 k_{1}+k_{2}\right) e_{1}+\left(k_{1}+k_{2}\right) w_{3}-k_{1} k_{2} w_{2}+k_{1}^{3} e_{1}-\left(k_{1}^{2}+k_{2}^{2}\right) w_{2}+u\right] \\
& +e_{\alpha}(-\dot{\hat{\alpha}})+e_{\beta}(-\dot{\hat{\beta}})+e_{\gamma}(-\dot{\hat{\gamma}})+e_{a}(-\dot{\hat{a}})+e_{b}(-\hat{\hat{b}}) .
\end{aligned}
$$


We choose the controller

$$
\begin{aligned}
u_{3}= & -2 w_{2}-\alpha x_{1}-\hat{\alpha} e_{1}+\beta x_{2}+\hat{\beta} e_{2}+\gamma x_{3}+\hat{\gamma} e_{3}+y_{1}^{3}-a y_{3}+\hat{a} e_{3}-x_{2}+\hat{b}-e^{x_{1}} \\
& +\left(2 k_{1}+k_{2}\right) e_{1}-\left(k_{1}+k_{2}\right) w_{3}+k_{1} k_{2} w_{2}+\left(k_{1}^{2}+k_{2}^{2}\right) w_{2}-k_{1}^{3} e_{1}-k_{3} w_{3},
\end{aligned}
$$

and the parameters are updated by the update law

$$
\begin{aligned}
\dot{\hat{\alpha}} & =w_{3} e_{1}+k_{4} e_{\alpha}, \dot{\hat{\beta}}=-w_{3} e_{2}+k_{5} e_{\beta}, \\
\dot{\hat{\gamma}} & =-w_{3} e_{3}+k_{6} e_{\gamma}, \dot{\hat{\hat{a}}}=-w_{3} e_{3}+k_{7} e_{a}, \text { and } \\
\dot{\hat{b}} & =-w_{3}+k_{8} e_{b} .
\end{aligned}
$$

Then we obtain

$$
\dot{V}_{3}=-k_{1} e_{1}^{2}-k_{2} w_{2}^{2}-k_{3} w_{3}^{2}-k_{4} e_{\alpha}^{2}-k_{5} e_{\beta}^{2}-k_{6} e_{\gamma}^{2}-k_{7} e_{a}^{2}-k_{8} e_{b}^{2} .
$$

Thus $\dot{V}_{3}$ is negative definite function.

Thus by a Lyapunov stability theory [50], the error dynamics (6.4) is globally exponentially stable and satisfied for all initial conditions.

Hence, the states of the master and slave systems are globally and asymptotically synchronized. Hence, we obtain the following result.

Theorem 6.1. The WINDMI chaotic system (6.1) and Coullet chaotic system (6.2) are globally and asymptotically synchronized with adaptive backstepping conrol

$$
\begin{aligned}
u_{3}= & -2 w_{2}-\alpha x_{1}-\hat{\alpha} e_{1}+\beta x_{2}+\hat{\beta} e_{2}+\gamma x_{3}+\hat{\gamma} e_{3}+y_{1}^{3}-a y_{3}+\hat{a} e_{3}-x_{2}+\hat{b}-e^{x_{1}} \\
& +\left(2 k_{1}+k_{2}\right) e_{1}-\left(k_{1}+k_{2}\right) w_{3}+k_{1} k_{2} w_{2}+\left(k_{1}^{2}+k_{2}^{2}\right) w_{2}-k_{1}^{3} e_{1}-k_{3} w_{3},
\end{aligned}
$$

by using adaptive parameter update law

$$
\begin{aligned}
\dot{\hat{\alpha}} & =w_{3} e_{1}+k_{4} e_{\alpha}, \dot{\hat{\beta}}=-w_{3} e_{2}+k_{5} e_{\beta}, \\
\dot{\hat{\gamma}} & =-w_{3} e_{3}+k_{6} e_{\gamma}, \dot{\hat{a}}=-w_{3} e_{3}+k_{7} e_{a}, \text { and } \\
\dot{\hat{b}} & =-w_{3}+k_{8} e_{b} .
\end{aligned}
$$

where $k_{i}, i=1,2,3, \ldots, 11$. are positive constants.

\subsection{Numerical simulation}

For the numerical simulations, the fourth order Runge-Kutta method is used to solve the differential equations (6.1) and (6.2) with the feedback controls $u$.

The parameters of the systems (6.1) and (6.2) are taken in the case of chaotic case as

$$
a=0.7, b=2.5
$$

and

$$
\alpha=5.5, \beta=3.5, \gamma=1 \text {. }
$$


The initial value of the drive system (6.1) are chosen as

$$
x_{1}(0)=0.341, x_{2}(0)=0.598, x_{3}(0)=0.928
$$

and response system (6.2) are chosen as

$$
y_{1}(0)=792, y_{2}(0)=0.734, y_{3}(0)=0.253 \text {. }
$$

The initial values of the parameter estimates are taken as:

$$
\hat{\alpha}(0)=1.2, \hat{\beta}(0)=1.5, \hat{\gamma}(0)=3, \hat{a}(0)=8, \hat{b}(0)=4 .
$$

We take the parameters $k_{i}=2, i=1,2,3 \ldots, 11$.

Figure 9 depict the synchronization of WINDMI and Coullet chaotic systems (6.1) and (6.2).

Figure 10 depict the synchronization error between WINDMI and Coullet chaotic systems (6.1) and (6.2).

Figure. 11 shows the estimated values of the parameters $\hat{a}, \hat{b}$ and $\hat{c}$ converges to system parameters $\alpha=5.5, \beta=3.5, \gamma=1, a=0.7$, and $b=2.5$.

\section{Conclusion}

In this paper, adaptive backstepping control method has been applied to estimate the fixed but unknown parameter and achieve global chaos synchronization for WINDMI and Coullet chaotic systems. Since the Lyapunov exponents are not required for these calculations, the adaptive backstepping control design is very effective and convenient to achieve global chaos synchronization. Numerical simulations have been given to illustrate and validate the effectiveness of the adaptive backstepping control based synchronization schemes of the WINDMI and Coullet chaotic systems.

\section{References}

[1] H. Fujisaka and T. Yamada, Stability theory of synchronized motion in coupledoscillator systems, Progress of Theoretical Physics, 63(1983), 32-47.

[2] L. M. Pecora and T. L. Carroll, Synchronization in chaotic systems, Phys. Rev. Lett., 64(1990).

[3] L. M. Pecora and T. L. Carroll, Synchronizing chaotic circuits, IEEE Trans. Circ. Sys., 38(1991), 453-456.

[4] K. T. Alligood, T. Sauer and J. A. Yorke, Chaos: An Introduction to Dynamical Systems, Berlin, Germany: Springer-Verlag, 1997.

[5] Edward Ott, Chaos in Dynamical Systems, United Kingdom, Cambridge University Press, 2002. 
[6] Y. M. Wang and H. Zhu, Generalized synchronization of continuous chaotic systems, Chaos, Solitons and Fractals, 27(2006), 97-101.

[7] Z. M. Ge and C. C. Chen Phase synchronization of coupled chaotic multiple time scales systems, Chaos, Solitons and Fractals, 20(2004), 639-647.

[8] J. Qiang, Projective synchronization of a new hyper chaotic Lorenz systems, Phys. Lett. A, 370(2007), 40-45.

[9] Y. Jian-Ping and L. Chang-Pin, Generalized projective synchronization for the chaotic Lorenz systems and the chaotic Chen system, Journal of Shanghai University, 10(2006), 299-304.

[10] R. H. Li, W. Xu and S. Li, Adaptive generalized projective synchronization in different chaotic systems based on parameter identifiction, Phys. Lett. A, 367(2007), 199-206.

[11] V. Sundarapandian and P. Sarasu, Generalized projective synchronization of doublescroll chaotic systems using active feedback control, CCSIT 2012, Part-I, LNICST 84, Springer Heldelberg, Dordrecht, London, Newyork, pp. 111-118, 2012. Edited by N. Meganathan et., al.

[12] P. Sarasu, and V. Sundarapandian, Generalized projective synchronization of threescroll chaotic systems via. active control, CCSIT 2012, Part-I, LNICST Vol. 84, Springer Heldelberg, Dordrecht, London, Newyork, pp. 124-133, 2012. Edited by N. Meganathan et., al.

[13] V. Sundarapandian and S. Sivaperumal, Anti-synchronization of hyperchaotic Lorenz and hyperchaotic Chen systems by adaptive control, International Journal of Automation and Computing, 9(2012), 274-279.

[14] V. Sundarapandian and R. Karthikeyan, Anti-synchronization of four wing chaotic systems via sliding mode control, International Journal of Signal System Control and Engineering Application, 4(2011), 18-25.

[15] K. Murali and M. Lakshmanan, Secure communication using a compound signal using sampled-data feedback, Applied Mathematics and Mechanics, 11(2003), 1309-1315.

[16] T. Yang, and L. O. Chua Generalized synchronization of chaos via linear transformations, Internat. J. Bifur. Chaos, 9(1999), 215-219.

[17] K. Murali and M. Lakshmanan, Chaos in Nonlinear Oscillators: Controlling and Synchronization, Singapore: World Scientific, 1996.

[18] S. K. Han, C. Kerrer, and Y. Kuramoto, D-phasing and bursting in coupled neural oscillators, Phys. Rev. Lett., 75(1995), 3190-3193.

[19] B. Blasius, A. Huppert and L. Stone, Complex dynamics and phase synchronization in spatially extended ecological system, Nature, 399(1999), 354-359.

[20] L. Kocarev, and U. Parlitz, General approach for chaotic synchronization with applications to communications, Phys. Rev. Lett., 74(1995), 5028-5030.

[21] Zuolei Wang, Chaos synchronization of an energy resource system based on linear control, Nonlinear Analysis: Real world Application,(article in press).

[22] Jiang Wang, Lisong Chen, Bin Deng, Synchronization of ghostburster neurons in external electrical stimulation via $H_{\infty}$ variable universe fuzzy adaptive control, Chaos, Solitons and Fractals, 39(2009), 2076-2085. 
[23] F. M. Moukam Kakmeni, J. P. Nguenang, and T. C. Kofane, Chaos synchronization in bi-axial magnets modeled by bloch equation, Chaos, Solitons and Fractals, 30(2006), 690-699.

[24] J. L. Hindmarsh and R. M. Rose, A model of neuronal bursting using 3-coupled 1 st order differential equations, Proc. Roy. Soc. Lond. B. Biol, 221(1984), 81-102.

[25] Yan-Qiu Che, Jiang Wang, Kai-Ming Tsang and Wai-Lok Chen, Unidirectional synchronization for Hindmarsh-Rose neurons via robust adaptive sliding mode control, Nonlinear Analysis: Real world Application, 11(2010), 1096-1104.

[26] Guang Zhao Zeng, Lan Sun Chen and Li Hua Sun, Complexity of an SIR epidemic dynamics model with impulsive vaccination control, Chaos, Solitons and Fractals, 26(2005), 495-505.

[27] Junxa Wang, Dianchen Lu and Lixin Tian, Global synchronization for time delay WINDMI system, Chaos, Solitons and Fractals, 30(2006), 629-635.

[28] W. Horton, R. S. Weigel and J. C. Sprott, Chaos and the limits of predictability for the solar-wind-driven magnetosphere-ionosphere system, Physics of Plasmas, 8(2003), $2946-2952$.

[29] W. Horton and J. Doxas, A low-dimentional dynamical model for the solar wind driven geotail-ionosphere system, Journal of Geophysical Research A, 103(1998), 4561-4512.

[30] J. P. Smith, J. L. Thiffeault and W. Horton, Dynamical range of WINDMI model: an exploration of possible magnetospheric plasma states, Journal of Geophysical Research A, 105(2000), 12983-12996.

[31] E. Ott, C. Grebogi and J. A. Yorke, Controlling chaos, Phys. Rev. Lett., 64(1990), 1196-1199.

[32] J. H. Park, and O. M. Kwon, A novel criterion for delayed feedback control of timedelay chaotic systems, Chaos, Solitons and Fractals, 17(2003), 709-716.

[33] H. T. Yau, Design of adaptive sliding mode controller for chaos synchronization with uncertainties, Chaos, Solitons and Fractals, 22(2004), 341-347.

[34] V. Sundarapandian, Global chaos synchronization of the Pehlivan systems by sliding mode control, International J. Computer Science and Engineering, 03(2011), 21632169.

[35] V. Sundarapandian and R. Suresh, Global chaos synchronization for Rossler and Arneodo chaotic systems by nonlinear control, Far East Journal of Applied Mathematics, 44(2010), 137-148.

[36] V. Sundarapandian and R. Suresh, New results on the global chaos synchronization for Liu-Chen-Liu and Lü chaotic systems, PEIE 2010, CCIS Vol. 102, Springer-Verlag Berlin Heidelberg, pp. 20-27, 2010. Edited by V. V. Das, J. Stephen et., al.

[37] X. Wu and J. L, Parameter identification and backstepping control of uncertain Lu system, Chaos, Solitons and Fractals, 18(2003), 721-729.

[38] Y. G. Yu and S. C. Zhang, Adaptive backstepping synchronization of uncertain chaotic systems, Chaos, Solitons and Fractals, 27(2006), 1369-1375.

[39] R. Suresh and V. Sundarapandian, Global chaos synchronization of WINDMI and Coullet chaotic systems using backstepping control, Far East Journal of Mathematical Sciences, 67(2012), 265-287. 
[40] R. Suresh and V. Sundarapandian, Hybrid synchronization of n-scroll Chua and Lur'e chaotic systems using backstepping control via novel feedback, Archives of Control Sciences, 22(LVIII)(2012), 255-278.

[41] R. Suresh and V. Sundarapandian, Synchronization of n-scroll hyperchaotic Chua circuit using backstepping control with recursive feedback, Far East Journal of Mathematical Sciences, 73(2013), 73-95.

[42] J. Lu, X. Wu, X. Han, and J. Lu, Adaptive feedback synchronization of a unified chaotic system, Phys. Lett. A, 329(2004), 327-333.

[43] J. H. Park, S. M. Lee and O. M. Kwon, Adaptive synchronization of Genesio-Tesi chaotic system via a novel feedback control, Physics Letters A, 371(2007), 263-270.

[44] J. H. Park, Adaptive control for modified projective synchronization of a fourdimensional chaotic system with uncertain parameters, J. Computational and Applied Math., 213(2008), 288-293.

[45] Min Xiao, Jinde Cao, Synchronization of a chaotic electronic circuit system with cubic term via adaptive feedback control, Communication in Nonlinear Science and Numerical simulation, 14(2009), 3379-3388.

[46] Yan-Wu Wang, Changyun Wen, Meng Yang and Jiang- Wen Xiao, Adaptive control and synchronization for chaotic systems with parametric uncertainities, Phys. Lett. A., 372(2008), 2409-2414.

[47] Zhiyong Ye and Cunbing Deng, Adaptive synchronization to a general nonautonomous chaotic system and it applications Nonlinear Analysis: Real World Applications, 13(2012), 840-849.

[48] J. C. Sprott, Chaos and Time- Series Analysis, Oxford University Press, New York, USA, 2003.

[49] P. Coullet, C. Tresser and A. Arneodo, Transition to stochasticity for a class of forced oscillators, Phys. Lett. A, 72(1979), 268-270.

[50] W. Hahn, The Stability of Motion, Berlin, Germany: Springer-Verlag, 1967. 


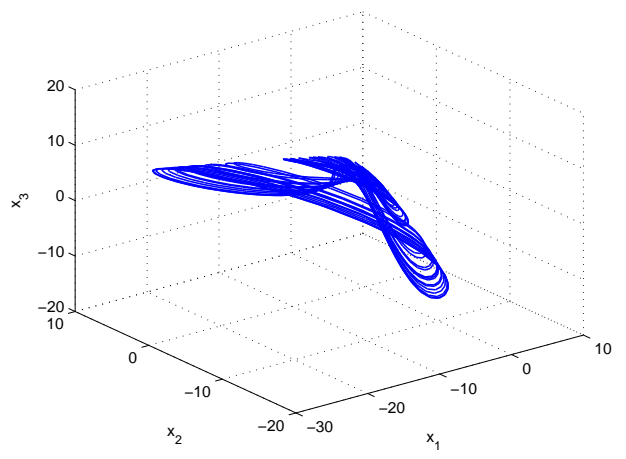

Figure 1: Phase orbit of WINDMI chaotic system

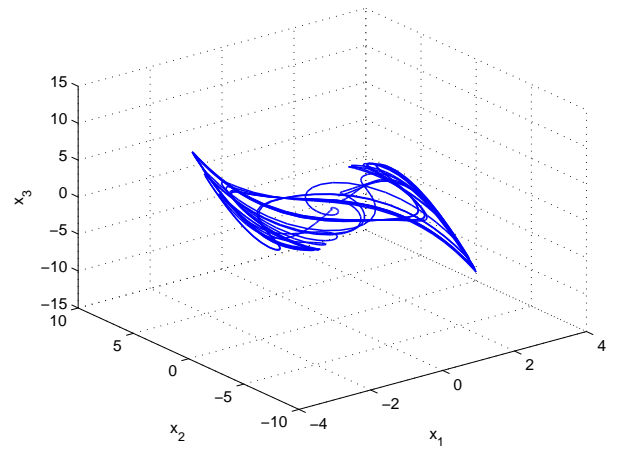

Figure 2: Phase orbit of Coullet chaotic system 

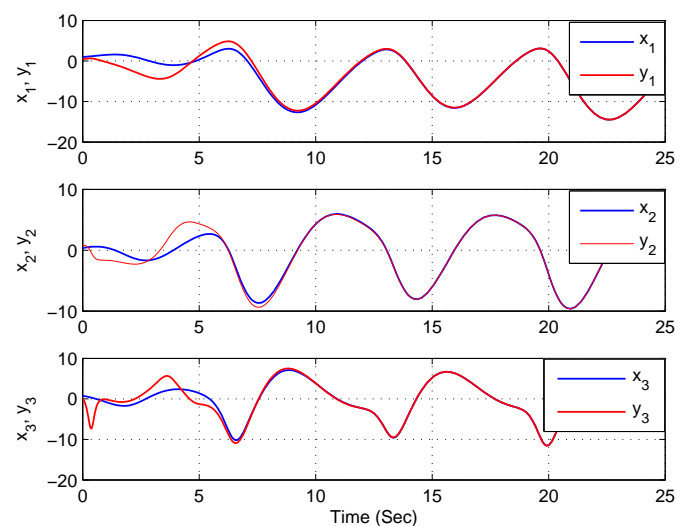

Figure 3: Synchronization of Identical WINDMI Chaotic Systems

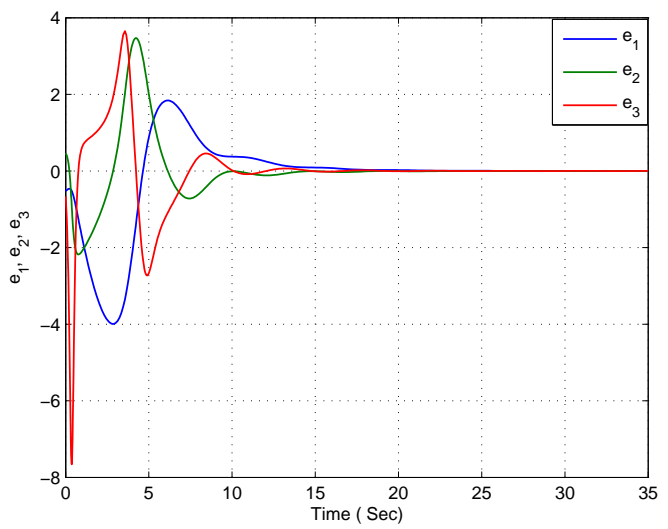

Figure 4: Error portrait of Identical WINDMI Chaotic Systems 


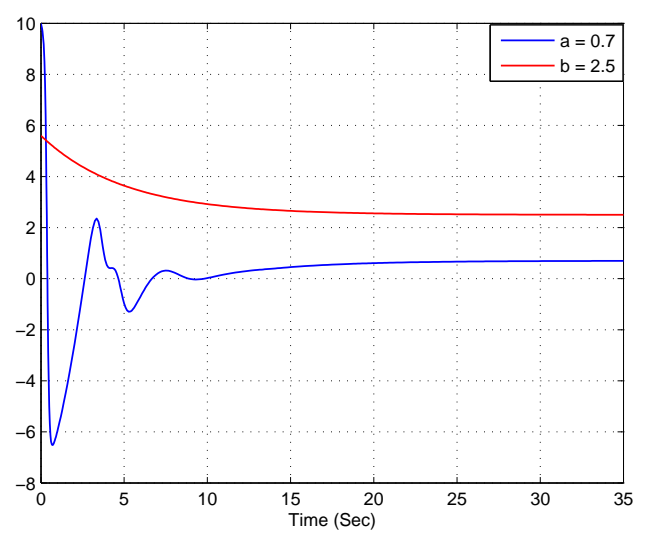

Figure 5: Parameter estimates of $\hat{a}$ and $\hat{b}$
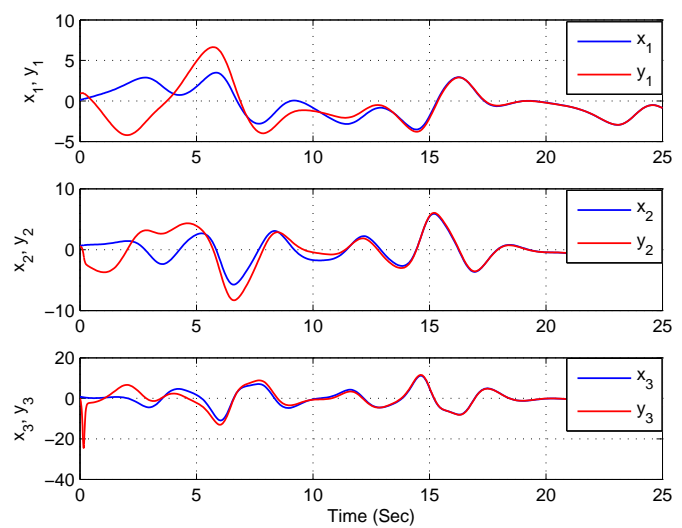

Figure 6: Synchronization of Identical Coullet Chaotic Systems 


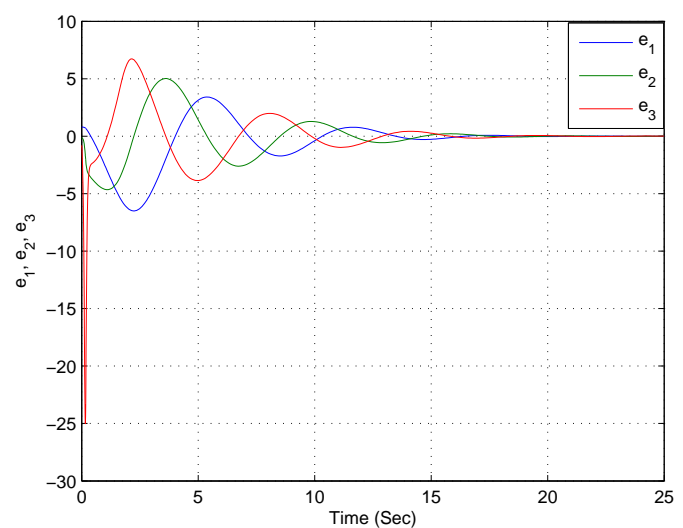

Figure 7: Error portrait of Identical Coullet Chaotic Systems

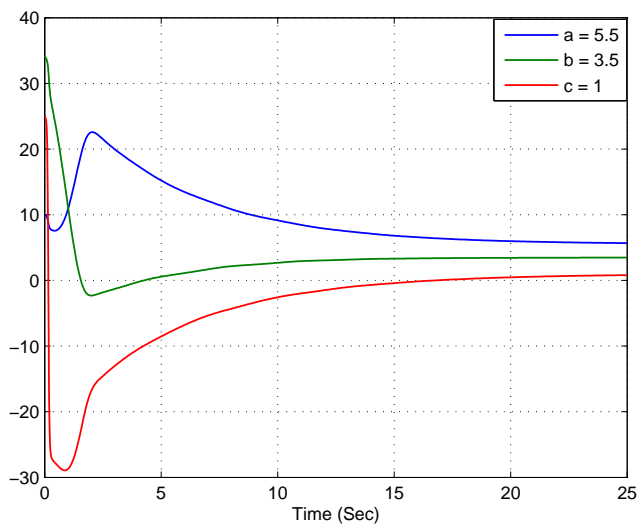

Figure 8: Parameter estimates of $\hat{a}, \hat{b}$ and $\hat{c}$ 

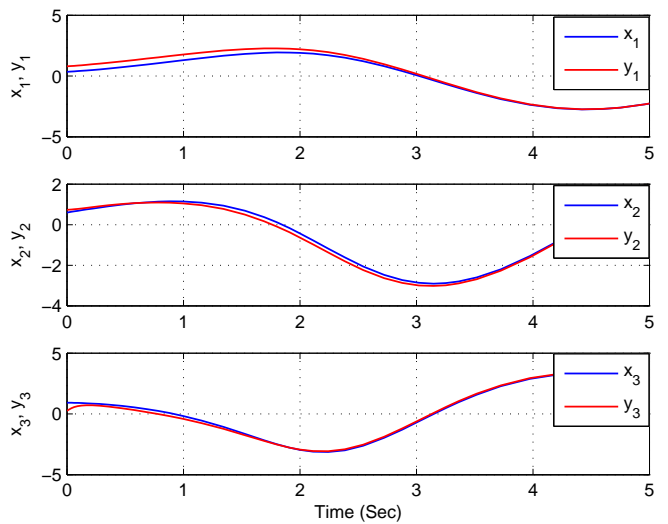

Figure 9: Synchronization of WINDMI and Coullet Chaotic Systems

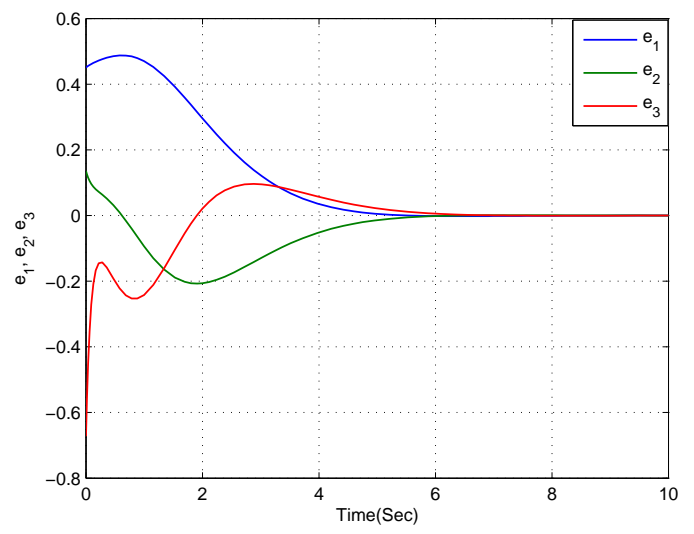

Figure 10: Error portrait of WINDMI and Coullet Chaotic Systems 


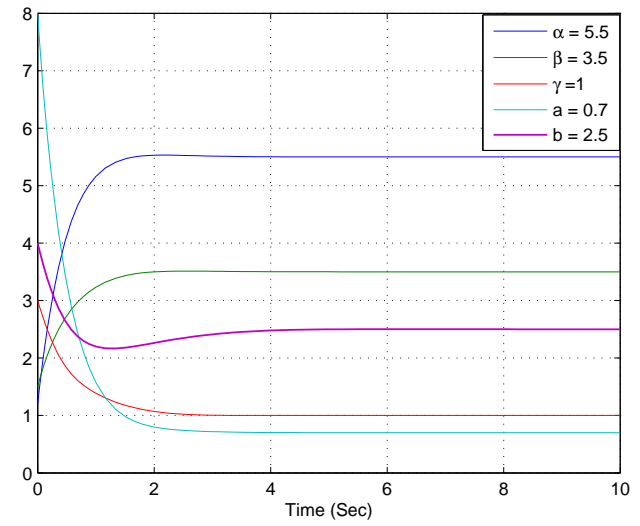

Figure 11: Parameter estimates of $\hat{\alpha}, \hat{\beta}, \hat{\gamma}, \hat{a}$ and $\hat{b}$ 